\title{
III. Tschechisch-slowakisches Symposium der Geschichte der Medizin, Pharmazie und Veterinärmedizin mit internationaler Beteiligung
}

Vom 25. bis zum 27. Juni 1996 trafen sich zum dritten Male Historiker der Medizin, der Pharmazie und der Veterinärmedizin in Benešov bei Prag zum Tschechisch-slowakischen Symposium, an dem auch Wissenschaftler aus Slowenien, Ungarn, Österreich und Deutschland teilnahmen. Veranstaltet vom Institut für Geschichte der Medizin an der Medizinischen Fakultät der Prager Karlsuniversität, welches von Prof. Dr. Dr. Eugen Strouhal geleitet wird, bildete das reichhaltige Programm mit mehr als 80 Vorträgen ein wahres Florilegium. Nach einer Eröffnungssitzung im Plenum tagte man am ersten Tag in Sektionen, welche den Themen „Geschichte der gegenseitigen Beziehungen der Ärzte, Pharmazeuten und Veterinäre aus den Böhmischen Ländern, der Slowakei und Österreich“" sowie „Gesundheitswesen und Gesellschaft im 19. und 20. Jahrhundert" gewidmet waren. Den Großteil des Programmes (am zweiten und am dritten Tag in jeweils drei Parallelsitzungen) nahmen freie Themen ein, welche von biographischen und institutionsgeschichtlichen Studien bis zu Berichten über pathologisch-anatomische historische Untersuchungen reichten. Zu letzteren sollen nur die Vorträge von E. Strouhal, „Auf den Spuren der Variola-Epidemie bei den Mitgliedern der ägyptischen Königsfamilie der 20. Dynastie“, und von L. Horácková und L. Benešová, „French War-time Surgeons at the Battle of Austerlitz", genannt werden.

Es wurde bekanntgegeben, daß das IV. Symposium 1998 in Bratislava (Preßburg) stattfinden soll.

In der landschaftlich reizvollen Umgebung von Benešov, in unmittelbarer Nachbarschaft des Jagdschlosses Konopište mit seinem schönen Park, ergab sich auch außerhalb des Programmes Gelegenheit zum Meinungsaustausch und dem Anbahnen wissenschaftlicher Kontakte. Neben der Teilnahme an einer kulturhistorischen Stadtführung in Benešov, dem Besuch von Schloß Konopište und einem abendlichen Konzert in der St. Annen-Kirche hatte man die Möglichkeit, sich im AnschluB an das Symposium die wissenschaftshistorisch interessanten Schätze im benachbarten Prag anzusehen.

Auch an dieser Stelle sei Prof. Dr. Dr. Strouhal und seinen Mitarbeiterinnen und Mitarbeitern für die perfekte Organisation (es gab sogar Simultanübersetzung) und die liebenswürdige Gastfreundschaft gedankt.

Ingrid Kästner (Leipzig)

\section{Berichte vom Deutschen Wissenschafts- historikertag 1996}

Der Sitzungsabschnitt Krankheitsursachen und medizinische Wissenschaft 1880-1920-Konzeptionen, Argumentationen und Institutionen im Wandel versuchte aufzuzeigen, wie sich im genannten Zeitraum durch die modernen Erkenntnisse der naturwissenschaftlich orientierten Medizin kausales Denken in den experimentellen Ansätzen der Forschung im Sinne eines Paradigmenwechsels durchsetzte und große Hoffnungen in die Diagnostik und Therapie bisher gar nicht oder nur symptomatisch behandelbarer Krankheiten trug. Dabei wurde zunächst zum Teil sehr radikal mit traditionellen, nicht selten auf empirischer Basis multifaktoriell ausgerichteten ätiologischen Modellen gebrochen, was nicht nur Fortschritt, sondern auch Reduktionismus bedeutete, so daß später mit z.T. überraschenden, weil ausgerechnet konsequent kausalen Argumenten multifaktorielle Betrachtungsweisen wieder Eingang in die Medizin fanden, die eine realistischere Abbildung der Verursachungsmechanismen von Krankheit und damit auch umfassendere therapeutische Möglichkeiten inbegriffen.

NTM N.S. $5(1997)$ 